TAMKANG JOURNAL OF MATHEMATICS

Volume 23, Number 2, Summer 1992

\title{
ON GRAPH REPRESENTATION FOR CO-DIAGONAL GROUPS
}

\author{
WU-YEN LEE
}

\begin{abstract}
For a co-diagonal Butler group $G$ with co-representing graph, we examine the conditions under which pure subgroups and torsion-free quotients of $G$ have quasi-co-representing graphs.
\end{abstract}

\section{Introduction}

For a rank 1 torsion-free abelian group $X$, let type $(X)$ denote the isomorphism class of $X$ called a type. For any element $g$ of a finite rank torsionfree group $G$, we let $\operatorname{type}_{G}(g)$ denote the type $\left(\langle g\rangle_{*}\right)$, where $\langle g\rangle_{*}$ is the rank-1 pure subgroup generated by $g$. If $\tau$ is a type, let $G(\tau)=\{x \in$ $G^{\prime} \mid$ type $\left._{G}(x) \geq \tau\right\}$ and $G^{*}(\tau)=\Sigma\{G(\sigma) \mid \sigma>\tau\} . G[\tau]$ is defined to be $\cap$ $\left\{\operatorname{ker} f \mid f \in \operatorname{Hom}\left(G, X_{\tau}\right)\right\}$, where $X_{\tau}$ is a subgroup of $Q$ with type $\left(X_{\tau}\right)=\tau$. Also, define $G^{*}[\tau]=\cap\{G[\sigma] \mid \sigma<\tau\}$. An exact sequence of torsion-free abelian groups $0 \rightarrow A \rightarrow B \rightarrow C \rightarrow 0$ is co-balanced if $0 \rightarrow A / A[\tau] \rightarrow B / B[\tau]$ is pure exact for each type $\tau$.

Butler groups are pure subgroups of finite rank completely decomposable groups. This class of Butler groups plays an important role in the theory of finite-rank torsion-free abelian groups. In this paper we shall be concerned with co-diagonal groups.

Co-diagonal Butler groups are groups which are quasi-isomorphic to $G<$ $A\rangle=\left(A_{1} \oplus \ldots \oplus A_{n}\right) /\langle(1, \ldots, 1)\rangle_{*}$ for some $n$-tuple $A=\left(A_{1}, \ldots, A_{n}\right)$ of subgroups of $Q$. The group $G\langle A\rangle$ is the dual of $G(A)=\left\{\left(a_{1}, \ldots, a_{n}\right) \in\right.$

Received May 1, 1990. 
$\left.A_{1} \oplus \ldots \oplus A_{n} \mid \Sigma_{i=1}^{n} a_{i}=0\right\}$ [5]. This class of co-diagonal groups is presented in [1] and some of their properties can interpreted through graph representation [2].

Let $\left(A_{1}, \ldots, A_{n}\right)$ be an $n$-tuple of subgroups of $Q$ and $T$ be a connected labelled graph with vertices $A_{1}, \ldots, A_{n}$ and each edge $i j$ in $T$ labelled by a type $\tau^{i j}=\operatorname{type}\left(A^{i j}=A_{i}+A_{j}\right)=\sup \left\{\operatorname{type}\left(A_{i}\right), \operatorname{type}\left(A_{j}\right)\right\}$.

The graph $\mathbb{T}$ is a co-representing graph for $\left.G=G<A_{1}, \ldots, A_{n}\right\rangle=\left(A_{1} \oplus\right.$ $\left.\ldots \oplus A_{n}\right) / X$ with $X=\langle(1, \ldots, 1)\rangle_{*}$ if

(1) $0 \rightarrow G \stackrel{\delta_{T}}{\longrightarrow} D_{T}$ is co-balanced exact.

(2) For each type $\tau, \delta_{T}^{\tau}(G / G[\tau])=G^{\tau} \oplus G^{*}\langle\tau\rangle$, where $D_{T}=\oplus\left\{A^{i j} \mid i j \in T\right\}, \delta_{T}\left(x_{i}+X\right)=\oplus\left\{x_{i j} \mid x_{i j}=x_{i}\right.$ if $i<j$ and $x_{i j}=-x_{i}$ if $j<i, i j \in T\}$ with $x_{i} \in A_{i}, G^{\tau}=\oplus\left\{A^{i j} \mid \tau^{i j}=\tau, i j \in T\right\}, G^{*}<\tau>=$ $\Sigma_{k} \delta_{T} *\left\langle\tau>\left(\left(A_{k}+X\right) / X\right)\right.$ with $\delta_{T} *<>\left(\left(A_{k}+X\right) / X\right)=\delta_{T}\left(\left(A_{k}+X\right) / X\right) \cap D_{T *<\tau>}$ and $T^{*}\langle\tau\rangle=\left\{i j \in T \mid \tau^{i j}<\tau\right\}$.

The graph $T$ is a quasi-co-representing graph for a Butler group $G$ if $T$ is a co-representing graph for some $\left.G<A_{1}, \ldots, A_{n}\right\rangle$ quasi-isomorphic to $G$.

In [2], we have developed a realization theorem for graph which are finite, connected, and with edges labelled by types. Furthermore, for each co-diagonal Butler group $G<A>$, we can construct its co-representing graph.

In the paper, we are going to examine the conditions under which pure subgroups and torsion-free quotients of groups with co-representing graphs have quasi-co-representing graph. In particular, if $G$ is a co-diagonal Butler group with co-representing graph, then $G(\tau), G[\tau],\left\langle G^{*}(\tau)\right\rangle_{*}, G / G[\tau]$ and $G^{*}[\tau] / G[\tau]$ have quasi-co-representing graphs for each type $\tau$.

\section{Main Results}

Suppose that $T$ is a co-representing graph for $G=G\left\langle A_{1}, \ldots, A_{n}\right\rangle$, and that $S$ is a subset of the set of edges of $T$. The equivalence relation on the vertices of $T$ determined by $S$ is defined by $i \approx i$, and for $i \neq j, i \approx j$ whenever $i$ and $j$ are connected by a path in $T \backslash S$. Define $M<S>=\left\{g \in G \mid \delta_{T}(g) \in D_{S}\right\}$. Note that $M<S>$ is a pure subgroup of $G$ which is isomorphic to $\delta_{T}(G) \cap D_{S}$. 
Theorem 2.1. Assume that $T$ is a co-representing graph for $G=G<$ $A_{1}, \ldots, A_{n}>, S \subset \mathbb{T}$, and $V_{1}, \ldots, V_{k}$ is the set of equivalence classes of vertices of $T$ determined by $S$. Then

(a) $M<S>\cong G<B_{1}, \ldots, B_{k}>$, where $B_{i}=\cap\left\{A_{j} \mid j \in V_{i}\right\}$

if $k>1$, and $M<S>$ is 0 if $k=1$.

(b) $G / M<S>\cong G_{1} \oplus \ldots \oplus G_{k}$, where $G_{i}=G\left\langle A_{j} \mid j \in V_{i}\right\rangle$ if $\left|V_{i}\right|>1$, and $G_{i}=0$ if $\left|V_{i}\right|=1$.

Proof. (a) First, observe that $M<S>=\left\{g=\left(a_{1}, \ldots, a_{n}\right)+X \in G \mid a_{t}=\right.$ $\left.a_{t}, \in B_{i,}, t, t^{\prime} \in V_{i}, i=1, \ldots, k\right\}$.

To see this, let $g=\left(a_{1}, \ldots, a_{n}\right)+X \in G$. Then $\delta_{T}(g)=\oplus\left\{a_{i}+\left(-a_{j}\right) \mid i<\right.$ $j, i j \in T\}$. If $a_{t}=a_{t}, \in B_{i}$ for $t, t^{\prime} \in V_{i}, i=1, \ldots, k$, then the $i j$-coordunate $\left(\delta_{T}(g)\right)_{i j}$ of $\delta_{T}(g)$ is 0 for each $i j \in T \backslash S$ since $i$ and $j$ belong to the same class. Hence, $\delta_{T}(g) \in D_{S}$.

Conversely, if $\left(\delta_{T}(g)\right)_{i j}=0$ for each $i j \in T \backslash S$, then $a_{i}=a_{j}$ for each $i j \in T \backslash S$. Hence, $a_{t}=a_{t}$, for $t, t^{\prime} \in V_{i}, i=1, \ldots, k$.

Now define $f: G<B_{1}, \ldots, B_{k}>\rightarrow M<S>$ by $f\left(\left(b_{1}, \ldots, b_{k}\right)+X\right)=$ $\left(a_{1}, \ldots, a_{n}\right)+X$, where $a_{t}=b_{i}$ for $t \in V_{i}, i=1, \ldots, k$.

It is easy to see that $f$ is epic.

If $f\left(\left(b_{1}, \ldots, b_{k}\right)+X\right)=\left(a_{1}, \ldots, a_{n}\right)+X=0$, then $a_{1}=\ldots=a_{n}$. Hence, $b_{1}=\ldots=b_{k}$. and $\left(b_{1}, \ldots, b_{k}\right)+X=0$.

This proves that $f$ is monic.

Therefore, $f$ is an isomorphism and $G<B_{1}, \ldots, B_{k}>\cong M<S>$.

(b) we next show that $G / M<S>\cong G_{1} \oplus \ldots \oplus G_{k}$ by defining a function $h: G \rightarrow G_{1} \oplus \ldots \oplus G_{k}$, where $h\left(\left(a_{1}, \ldots, a_{n}\right)+X\right)=\oplus_{i=1}^{k}\left\{\left(a_{i_{1}}, \ldots, a_{i_{m}}\right)+X \mid i_{s} \in\right.$ $\left.V_{i}\right\}$. Then it is easy to see that $h$ is epic with $\operatorname{ker} h=M\langle S\rangle$. Hence, (b) is proved.

Examples of Theorem 2.1 are included in the following corollary.

Corollary 2.2. Suppose that $T$ is a co-representing graph for $G=G<$ $A_{1}, \ldots, A_{n}>$ and $\tau$ is a type. Then 
(a) $G(\tau)=M\left\langle S(\tau)>\right.$, where $S(\tau)=\left\{i j \in T \mid \tau^{i j} \geq \tau\right\}$.

(b) $G[\tau]=M<S[\tau]>$, where $S[\tau]=\left[i j \in T \mid \tau^{i j} \leq \tau\right\}$.

(c) $G(\tau), G[\tau],\left\langle G^{*}(\tau)\right\rangle_{*}, G / G[\tau]$ and $G^{*}[\tau] / G[\tau]$ have quasi-co-representing graphs induced by $T$.

Proof. Suppose that $g \in G$ and type $G(g) \geq \tau$. Then type $G(g)=$ type $_{D_{T}}$ $\left(\delta_{T}(g)\right)=\inf \left\{\operatorname{type}\left(A^{i j}\right) \mid \mathbb{\Pi}_{i j} \delta_{T}(g) \neq 0\right\} \geq \tau$. where $\mathbb{\Pi}_{i j}: \mathbb{D}_{T} \rightarrow A^{i j}$ is the projection of $D_{T}$ onto $A^{i j}$, noting that $0 \rightarrow G \stackrel{\delta_{T}}{\longrightarrow} D_{T}$ is co-balanced exact, hence pure exact. Hence, $\delta_{T}(g) \in \oplus\left\{A^{i j} \mid i j \in S(\tau)\right\}$.

Conversely, let $\delta_{T}(g) \in \oplus\left\{A^{i j} \mid i j \in S(\tau)\right\}$ for some $g \in G$. Then type ${ }_{D_{T}}$ $\left(\delta_{T}(g)\right) \geq \tau$, since type $\left(A^{i j}\right) \geq \tau$ for each $i j . \in S(\tau)$. Hence, type ${ }_{G}(g)=$ type $_{D_{T}}\left(\delta_{T}(g)\right) \geq \tau$ and $g \in G(\tau)$.

(b) First, note that $g[\tau]=\langle G(\sigma) \mid \sigma \notin \tau\rangle_{*}$, by Proposition 1.9 in [3]. Suppose that $g \in G$ and type $G(g) \not \tau$. Then type $G(g)=\operatorname{type}_{D_{T}}\left(\delta_{T}(g)\right)=$ $\inf \left\{\operatorname{type}\left(A^{i j}\right) \mid \Pi_{i j} \delta_{T}(g) \neq 0\right\} \mathbb{L} \tau$. Hence, $\delta_{T}(g) \in \oplus\left\{A^{i j} \mid i j \in S[\tau]\right\}$. Otherwise type $D_{T}\left(\delta_{T}(g)\right) \leq \tau$, because if type $\left(A^{i j}\right) \leq \tau$ for some $i j \in T$ with $\Pi_{i j} \delta_{t}(g) \neq 0$, then type $D_{T}\left(\delta_{T}(g)\right) \leq$ type $\left(A^{i j}\right) \leq \tau$.

For eaach $x \in G[\tau]$, there exists $0 \neq m \in Z$ such that $m x=g_{1}+\ldots+g_{k}$ with type ${ }_{G}\left(g_{i}\right) \& \tau$. Then $\delta_{T}(m x) \in \oplus\left\{A^{i j} \mid i j \in S[\tau]\right\}$, since each $\delta_{T}\left(g_{i}\right) \in$ $\oplus\left\{A^{i j} \mid i j \in S[\tau]\right\}$. It follows that $\delta_{T}(x) \in \oplus\left\{A^{i j} \mid i j \in S[\tau]\right\}$, because $\delta_{T}(G)$ is pure in $D_{T}$. This proves that $\delta_{T}(G[\tau]) \subset \oplus\left\{A^{i j} \mid i j \in S[\tau]\right\}$.

Conversely, if $\delta_{T}(g) \in \oplus\left\{A^{i j} \mid i j \in S[\tau]\right\}$, then $g \in G[\tau]$, since $\delta_{T}^{\tau}(G / G[\tau])=$ $G^{\tau} \oplus G^{*}<\tau>C \oplus\left\{A^{i j} \mid i j \in \mathbb{T}, \tau^{i j} \leq \tau\right\}$. The reason is that if $g \notin G[\tau]$, then $g+G[\tau] \neq 0$. It follows that $\delta_{T}^{\tau}(g+G[\tau])=\delta_{T}(g)+D_{T}[\tau] \neq 0$ in $D_{T} / D_{T}[\tau]=$ $\oplus\left\{A^{i j} \mid i j \in T \tau^{i j} \leq \tau\right\}$, noting that $0 \rightarrow G / G[\tau] \stackrel{\delta_{T}^{\tau}}{\longrightarrow} D_{T} / D_{T}[\tau]$ is pure exact. This means that there exists $i j \in T$ with $\tau^{i j} \leq \tau$ such that $\left(\delta_{T}(g)\right)_{i j} \neq 0$. Then $\delta_{T}(g) \notin \oplus\left\{A^{i j} \mid i j \in S[\tau]\right\}$, a contradiction.

(c) By (a), (b) and Theorem 2.1, $G(\tau)$ and $G[\tau]$ have quasi-co-representing graphs. It follows that $\left\langle G^{*}(\tau)\right\rangle_{*}=G(\tau)[\tau]$ has a quasi-co-representing graph.

To show that $G / G[\tau]$ has quasi-co-representing graph, it is sufficient to prove that if $V_{1}, \ldots, V_{k}$ are the equivalence classes of vertices determined by $S[\tau]$, then 
$\left|V_{i}\right|>1$ for at most one $i$. In this case, $G / G[\tau] \cong G<A_{j} \mid j \in V_{i}>$ has a quasi-co-representing graph by Theorem 2.1.(b). To see that this is indeed the case, note that $V_{1}=\{i \mid i j \in T \backslash S[\tau]$ for some $j\}$ is an equivalence class, since $T<\tau\rangle=\left\{i j \in T \mid \tau^{i j} \leq \tau\right\}$ is connected by Corollary 2.9.(a) in [2]. On the other hand, if $i \notin V_{1}$, then the equivalence class of $i$ is $\{i\}$, since $i$ does not lie on an edge of $T \backslash S[\tau]$.

Finally, $G / G[\tau]=G^{*}[\tau] / G[\tau] \oplus G^{\prime}$ where $G^{\prime} \cong G / G^{*}[\tau]$ and $G^{*}[\tau] / G[\tau]=$ $(G / G[\tau])(\tau)$ by Theorem 1.7. (c) in [3]. Hence, $G^{*}[\tau] / G[\tau]$ has a quasi-corepresenting graph.

Actually, $G^{*}[\tau] / G[\tau]$ is $\tau$-homogeneous completely decomposable by Theorem 1.7.(c) in [3], which belongs to $\Omega$ and has a quasi-co-representing graph by Theorem 2.7 in [2], where $\Omega$ is the class of all torsion free abelian groups quasi-isomorphic to Butler groups which are homomorphic images of completely decomposable groups with rank-1 kernels.

\section{References}

[1] W. Lee, "Co-diagonal Butler groups", Chinese Journal of Mathematics 17 (1989), 259271.

[2] W. Lee, "Graph representation for co-diagonal Butler groups", to appear.

[3] D. Arnold and C. Vinsonhaler, "Pure subgroups of finite rank completely decomposable groups \|", Abelian Group Theory, Lecture Notes in Math., Vol. 1006, Springer-Verlag, Berlin and New York (1983), 97-143.

[4] M. C. R. Butler, "A class of torsion-free abelian groups of finite rank", Proc. London Math. Soc. 15 (1965), 680-698.

[5] F. Richman, "An extension of the theory of completely decomposable torsion-free abelian groups", Trans. Amer. Math. Soc. 279 (1983), 175-185. 